\title{
Downstream-based Scheduling for Energy Conservation in Green EPONs
}

\author{
Shen Chen*, Ahmad R. Dhaini*, Pin-Han Ho* ${ }^{*}$, Basem Shihada ${ }^{\dagger}$, Gangxiang Shen ${ }^{\ddagger}$, and Chih-Hao Lin ${ }^{\S}$ \\ * Dept. of Electrical and Computer Engineering, University of Waterloo, Canada \\ \{s92chen, adhaini, p4ho\}@uwaterloo.ca \\ $\dagger$ Dept. of Computer Science, KAUST, KSA \\ $\ddagger$ Dept. of Electronic and Information Science, Suzhou University, China, gxshen@ suda.edu.cn \\ $\S$ Dept. of Information Management Science, Chongyuan University, Republic of China, chlin@cgu.edu.tw
}

\begin{abstract}
Maximizing the optical network unit's (ONU) sleep time is an effective approach for achieving maximum energy conservation in green Ethernet passive optical networks (EPONs). While overlapping downstream and upstream ONU transmissions can maximize the ONU sleep time, it jeopardizes the quality of service (QoS) performance of the network, especially for downstream traffic in case the overlapping is based on the upstream time slot. In this paper, we study the downstream traffic performance in green EPONs under the limited service discipline and the upstream-based overlapped time window. Specifically, we first derive the expected mean packet delay, and then present a closed-form expression of the ONU sleep time, setting identical upstream/downstream transmission cycle times based on a maximum downstream traffic delay requirement. With the proposed system model, we present a novel downstream bandwidth allocation scheme for energy conservation in green EPONs. Simulation results verify the proposed model and highlight the advantages of our scheme over conventional approaches.
\end{abstract}

Index Terms-Green EPON, Dynamic Bandwidth Allocation, Queuing Theory, Energy Efficiency.

\section{INTRODUCTION}

$\mathbf{E}$ Thernet Passive Optical Network (EPON) has been considered as one of the most attractive broadband access solutions. It comprises an Optical Line Terminal (OLT) that resides in a Central Office $(\mathrm{CO})$ and connects through a single fiber a set of Optical Network Units (ONUs) located at the customer premises. In recent years, EPON has been subject to extensive research efforts related to dynamic bandwidth allocation (DBA), quality-of-service (QoS) support, and lately energy efficiency.

To achieve efficient energy conservation in EPON, each ONU should be put into sleep mode as long as the predefined QoS constraints are not violated. To meet this goal, most previous studies manipulate DBA schemes such that an ONU can be put into sleep while the OLT is receiving data from other ONUs [1], [2], [3], [4]. Previous studies are based on a common assumption that the OLT can buffer the downstream traffic of a "sleeping" ONU until the ONU wakes up. Their common design goal is to minimize the ONU idle time as much as possible while allowing the OLT to schedule collision-free ONU transmissions over the shared media.

Two energy management mechanisms for EPONs have been reported in [2], namely Upstream Centric Scheduling (UCS) and Downstream Centric Scheduling (DCS). Using UCS, the OLT buffers the downstream traffic of an ONU and starts transmitting it only in the designated upstream time slot of the ONU so as to reduce the ONU active time. Conversely with DCS, the OLT launches downstream traffic to the ONU whenever available. Thus, an ONU switches to the active mode whenever it needs to transmit upstream traffic or to receive downstream traffic. Clearly, UCS saves more energy by overlapping the downstream and upstream transmissions, while DCS can achieve better QoS performance since the upstream and downstream transmissions are scheduled independently and hence can be manipulated separately.

Green Bandwidth Allocation (GBA) has been reported in [4] as an effective framework for energy saving in PON. It is featured with a hybrid cyclic/deep sleep mode enabled at the ONUs, batch-mode transmission at OLT and ONUs, and UCS-based bandwidth allocation. With GBA, the ONU goes into the sleep mode for a certain amount of time before waking up to send/receive a batch of buffered upstream/downstream traffic. Due to the batch mode transmission, an ONU can avoid frequent switching between on and off state in order to minimize the overhead time during the switching. The authors of [3] further developed an analytical formulation for the delay of upstream traffic based on which the maximum sleep time is derived for each ONU.

To the best of our knowledge, all the previously reported energy saving schemes based on the UCS protocol have only considered the upstream packet delay constraint to compute the maximum ONU sleep time; while the QoS constraints of downstream traffic has never been considered. Note that under UCS, the downstream transmission of an ONU occurs during the upstream transmission period, thus the downstream QoS may not be ensured. Therefore, when the downstream traffic is much heavier than the upstream traffic (which is very common in practical PON systems), the maximum sleep time of each ONU by merely analyzing the upstream direction using the upstream traffic delay constraint will be very far from accurate.

We envision the importance of a downstream scheduling scheme at the OLT that ensures the packet delay constraint for each ONU. Thus, the paper investigates the maximum sleep scheduling for both downstream and upstream directions in the green EPON design under the GBA framework. We firstly consider a generic scheduling mechanism by allowing 
each ONU to wake up and start downstream and upstream transmissions in a round-robin manner, in which a mean packet delay expression is derived with the limited service discipline. To the best of our knowledge, delay analysis based on the limited service discipline has only been reported in [5], strictly for upstream bandwidth allocation due to its complexity. Hence, the novelty of our work is that we consider it for downstream bandwidth allocation, and for ONU sleep time computation. Moreover, in legacy EPONs, the downstream traffic is broadcast in nature, and hence downstream scheduling has never been considered as a research problem before; whereas in terms of energy conservation, it is considered as a main research challenge for the design of green EPONs [3].

With the proposed analytical model, we develop a novel scheduling algorithm, which runs at the OLT in order to compute the maximum ONU sleep time without violating the delay constraints for both the upstream and downstream transmissions. Extensive simulations are conducted to verify the proposed analytical model and highlight the effectiveness of the proposed scheduling algorithm.

The rest of the paper is organized as follows. Section II provides a survey on the related work in green EPONs. The system model is discussed in Section III. Section IV presents the downstream delay analysis along with the closed-form expressions of mean packet delay and maximum ONU sleep time. Section V presents the proposed energy efficient downstream traffic scheduling protocol. Simulation and numerical results are given in Section VI. Section VII concludes the paper and discusses our future work.

\section{RELATED WORK}

Although putting ONUs into sleep has been the most promising energy saving methods in PONs, an efficient and effective implementation of the ONU sleep mode is still subject to many open challenges. The study in [6] provided feasible implementations of the ONU sleep mode in PONs and discussed related issues such as ONU-OLT clock synchronization and QoS requirements of delay sensitive applications. With the introduction of fast clock recovery circuit in [7], energy can be saved in PONs using the proposed Just-In-Time (JIT) sleep control scheme, which allows the ONU to sleep within the DBA cycle (typically equal to $2 \mathrm{~ms}$ ). Note that the active period and the subsequent sleep period, including the overhead time that is due to the switching from sleep to active mode, form the ONU sleep cycle. It is clear that the sleep time in the sleep cycle at an ONU is at least the time when other ONUs are transmitting or receiving traffic. Thus the synchronization and traffic detection functions are essential. To ensure precise synchronization, the study in [8] proposed a fast ONU sleep mode which enables an ONU to automatically wake up and start transmitting/receiving traffic in the allocated time slot. In [9], a new Medium Access Control (MAC) protocol was presented for the ONUs to periodically wake up within the designated time slots when the system is operating at low network load. Here, the ONU can turn off its transmitter and receiver to reduce its power consumption. Although the study showed the potential to achieve energy saving by putting the ONUs into sleep, it does not mention how to determine the sleep time to achieve better performance. From the literature review in [3], it is clear that the previously reported studies on green PONs have neither provided a comprehensive approach to determine the maximum ONU sleep time, nor investigated the downstream packet scheduling problem, which is crucial for meeting the QoS constraints of downstream and upstream traffics.

\section{A. Overview of UCS-based GBA Framework}

The study in [3] investigated a UCS-based framework for achieving energy-efficient EPON design, based on a green bandwidth allocation (GBA) framework, which aims to maximize the ONU sleep time and reduce the total overhead time required to switch the ONU from the sleep mode to the active mode. Specifically, the authors proposed a batch mode-enabled transmission at the ONUs and OLT, which enlarges the DBA cycle so as to reduce the impact of the ONU on-off overhead. This is nonetheless at the expense of QoS impairment in terms of packet delay. To address this problem, the authors proposed a new analytical model to determine the optimal sleep time of each ONU based on a maximum upstream packet delay constraint. Simulation results demonstrated that GBA is able to achieve up to $90 \%$ energy saving compared to conventional legacy DBA schemes. The main advantage of the GBA framework in [3],[4] is that each ONU can have longer sleep than that with any other reported approach thanks to the developed analytical model and the batch mode transmission, which is vital in achieving the best energy saving without violating the QoS constraints. However, since the downstream QoS was not considered, a remaining challenge is to design a sleep control mechanism that meets the delay constraints of downstream traffic in addition to the ones considered for upstream traffic.

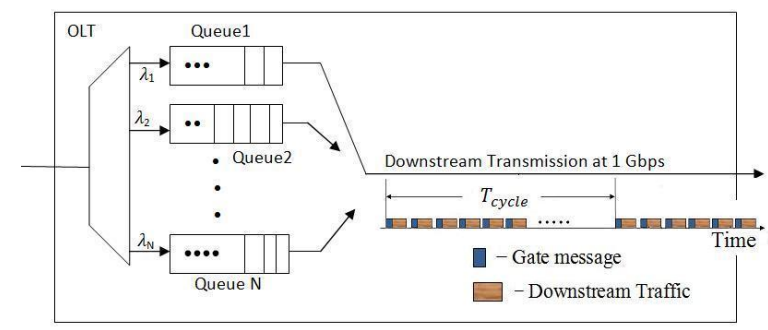

Fig. 1: The OLT model for the downstream traffic scheduling.

\section{System MODEL}

With UCS, the OLT and ONUs buffer the downstream and upstream traffic, respectively, and overlap their transmissions for a shorter (or longer) ONU active period (sleep time). By assuming the downstream traffic will be way heavier than that the upstream, the maximum sleep time should be determined according to the downstream traffic. Thus, it turns out to be an important problem in the evaluation of the downstream traffic buffer delay. Without loss of generality, we assume that the OLT is equipped with a downstream queue for each 
ONU. Fig. 1 shows the OLT system considered in this study, which can be taken as a queuing system and a multi-access channel, such that the ONUs queues are the "accessing" users. In other words, each queue has packets that are buffered and waiting to be transmitted to the corresponding ONU, and only one queue can have access to the shared media. Thus, it is feasible to take the channel as a common server accessed by the downstream queues. By considering the heterogeneous nature of downstream traffic, a fixed channel assignment that allocates fixed time slots or wavelength bands is not efficient. To schedule transmissions of several data streams into a statistical multiplexing system, the paper introduces a quasi-leaved polling model in which downstream traffic is scheduled so as to avoid collisions. We consider the quasileaved polling method because it allows the OLT to wait until all the REPORT messages (defined in the multi-point control protocol [MPCP]) are received before running the DBA. It is essential to the proposed scheduling algorithm which requires the complete knowledge of traffic demand of each ONU. Note that interleaved polling with adaptive cycle time (IPACT) [10] and any other version of interleaved polling schemes may have better network resource utilization, but they cannot enable general sleep time analysis since the OLT can only learn about one ONU's status at a time.

The OLT is the central controller of the network, which decides the scheduling of upstream and downstream transmissions for all the ONUs. Once a scheduling decision is made, MPCP's GATE control message is used to inform the ONU of both its allocated transmission size and scheduled sleep time within each DBA cycle. Let $T_{\text {cycle }}$ denote the periodic transmission cycle in which the OLT completes the downstream transmissions for all the ONUs, and without loss of generality let each ONU be served in a round-robin manner. With this regards, the upstream and downstream transmissions of the ONUs have an identical transmission cycle time $T_{\text {cycle }}$.

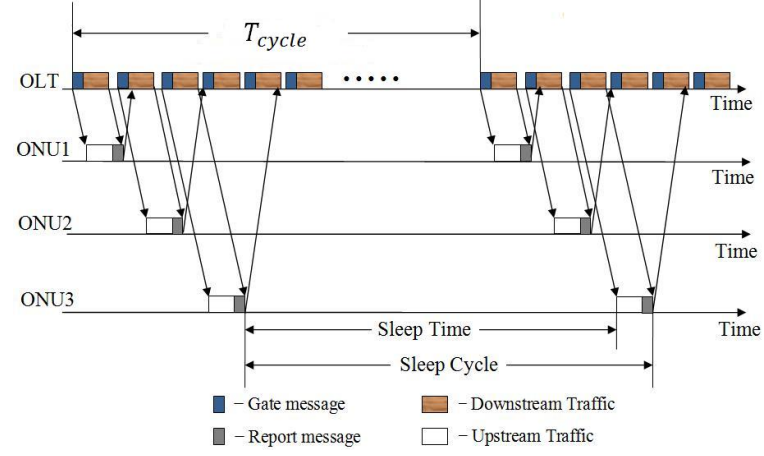

Fig. 2: Overlapped upstream and downstream traffic scheduling.

Each downstream queue at the OLT buffers the traffic destined for the corresponding ONU until the reserved downstream transmission time slot arrives, which is also used as the upstream transmission slot as shown in Fig. 2. Upon the arrival of the downstream time slot, the OLT transmits the data in queue $i$ to the corresponding ONU $i$. Note that $T_{\text {cycle }}$ in the figure may vary from one cycle to the other depending on the traffic load, the number of ONUs $(N)$, and the maximum transmission window size. The processing time of a GATE message plus the guard interval between two contiguous downstream queues serves as a fixed reservation interval, denoted as $\bar{V}$.

With each ONU being served in a round robin manner, the sleep cycle can be seen as the time for all the other ONUs to finish the channel access once. As mentioned earlier and as demonstrated in Fig. 2, the sleep cycle of ONU $i$ comprises three components: the sleep time, the active transmission time, and the overhead time required to switch the ONU from the sleep mode to the active mode. The overhead time accounts for the free running clock drifts, the ONU clock recovery time, and the synchronization to the network after recovering the OLT clock [8].

During the sleep mode, the ONU turns off both its transmitter and receiver so as to save energy. There are two main sleep modes defined in the gigabit-PON standard: deep sleep and fast sleep. In the former, the downstream or upstream traffic could be lost because all the ONU functions are shut down. In the latter, on the other hand, the ONU maintains the timing and traffic detection functions in order to wake up whenever new traffic arrives. With the proposed system model, the ONU goes into the sleep mode for a given amount of time (which is pre-scheduled and manipulatively calculated) before waking up to send/receive the buffered upstream/downstream traffic. Therefore, it allows an ONU to go into deep sleep and save the maximum energy during the sleep mode.

\section{Downstream Delay Analysis}

In this section, we analyze the mean packet delay of downstream traffic arriving at the OLT, which will then serve as an important basis for the determining the optimal ONU sleep time, as well as the proposed downstream scheduling scheme The notations used in the analysis are summarized in Table I.

We first start by modeling the system as a gated $N$ - user reservation system, in which the packets that arrive prior to the user's reservation interval are all transmitted, as shown in Fig. 3. Without loss of generality, to make our analysis tractable, we assume that downstream data packets arrive at the OLT according to a Poisson distribution at a mean rate of $\lambda / N$. Hence, the utilization factor of the total ingress traffic load is $\rho=\lambda \bar{X}$, such that $\bar{X}$ denotes the mean service time of each packet, and $\overline{X^{2}}$ is the second moment of service time. Let $\bar{V}$ and $\overline{V^{2}}$ denote the first two moments of the reservation interval, respectively. The corresponding packet delay analysis of a gated ${ }^{1}$ reservation system is given as follows [11]:

$$
\begin{aligned}
E(W) & =E(R)+E(Q)+E(Y) \\
& =\frac{\lambda \overline{X^{2}}}{2(1-\rho)}+\frac{(N+2-\rho) \bar{V}}{2(1-\rho)}+\frac{\sigma_{V}^{2}}{2 \bar{V}}
\end{aligned}
$$

where $E($.$) denotes the expected value of the operand. Hence,$ $E(W)$ denotes the expected packet delay in the queue, $E(R)$ is the expected mean residual time, $E(Q)$ is the expected

\footnotetext{
${ }^{1}$ Note that the gated service in EPON is different from the commonlyknown gated service in queueing theory. For more details on EPON's gated service, we refer the reader to [10].
} 
TABLE I: Notations used in the analysis

\begin{tabular}{|l|l|}
\hline Notation & Description \\
\hline $\mathrm{i}$ & the queue index and the related ONU index \\
\hline $\mathbb{D}_{\mathrm{i}, \xi}$ & $\begin{array}{l}\text { maximum allowable packet delay at ONU } i \\
\text { in the downstream transmission }\end{array}$ \\
\hline$S_{i}$ & Sleep cycle of ONU $i$ \\
\hline$T_{\mathrm{i}, \mathrm{d}}^{s}$ & downstream sleep time at ONU $i$ \\
\hline$T_{\mathrm{i}, \mathrm{d}}^{a}$ & $\begin{array}{l}\text { active period of the downstream transmission } \\
\text { at ONU } i\end{array}$ \\
\hline$T_{\mathrm{i}, \mathrm{d}}^{o}$ & overhead time at ONU $i$ for downstream \\
\hline$X_{\mathrm{i}, \xi}$ & service time of packet $\xi$ to ONU $i$ \\
\hline$\overline{X_{\mathrm{i}, \xi}}$ & mean service time of packet $\xi$ to ONU $i$ \\
\hline$\overline{X_{\mathrm{i}, \xi}^{2}}$ & the second moment of service time \\
\hline$\overline{W_{\mathrm{i}, \xi}}$ & $\begin{array}{l}\text { the mean packet queuing delay destined for } \\
\text { ONU } i\end{array}$ \\
\hline$D_{\mathrm{i}, \xi}$ & total delay of a packet to ONU $i$ \\
\hline$T_{\mathrm{prop}}$ & Packet propagation time \\
\hline$\xi$ & a packet of the downstream transmission \\
\hline$\lambda_{\mathrm{i}}$ & arrival packet rate to queue $i$ at OLT \\
\hline$\overline{Q_{\mathrm{i}}}$ & the average queue size of queue $i$ at OLT \\
\hline$C$ & utilization factor of queue $i$ at OLT \\
\hline$\rho_{\mathrm{i}}$ & the line \\
\hline$\rho$ & \\
\hline
\end{tabular}

mean service delay, $E(Y)$ is the expected mean reservation delay, and $\sigma_{V}^{2}$ is the variance of each reservation time. For consistency, the expected value notation will be replaced by the mean value one (e.g., $E(Y)$ will be referred to as $\bar{Y}$ ).

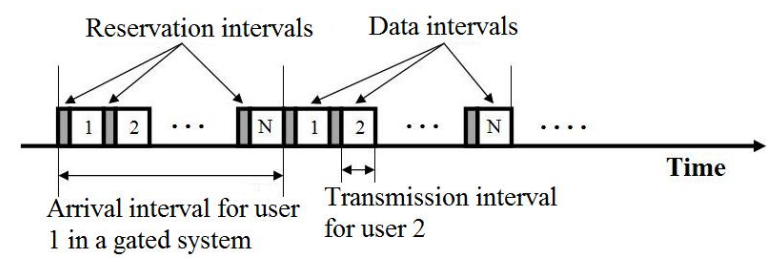

Fig. 3: Downstream traffic scheduling with $\mathrm{N}$ queues at the OLT.

The following analysis is based on the limited service discipline, which is the bulk of our work. Let the maximum transmission window be denoted as $T^{\max }$, and the maximum number of bytes transmitted in $T^{\max }$ be denoted as $W^{\max }$. Let the probability that a reservation interval is followed by a data interval of size $W^{\max }$ in steady-state be denoted as $P$, and the requested window size (in bytes) that is less than $W^{\max }$ be denoted as $\nu$. Consequently, the time required to transmit $\nu$ bytes is given as $T^{\nu}$. Clearly we have $T^{\max }=W^{\max } / C$ and $T^{\nu}=\nu / C$.

The ratio between data intervals and reservation intervals can be expressed as:

$$
\frac{\rho}{1-\rho}=\frac{P W^{\max }}{C \bar{V}} .
$$

Thus,

$$
P=\frac{C \bar{V} \rho}{W^{\max }(1-\rho)}
$$

Lemma 4.1: The OLT can only transmit $W^{\max }$ bytes for each queue per downstream cycle, thus the additional mean reservation delay for a $N$-user reservation system with the limited service discipline can be expressed as:

$$
\Delta Y=\frac{\lambda \bar{W} \bar{V} \bar{X} C P}{W^{\max }} .
$$

Proof: Let $€$ be the total queue size at the OLT (of all queues). Hence the mean queue size is $\mathrm{\iota} / N$. Using Little's Law, we have $€=\lambda \bar{W}$. Furthermore, using the limited service discipline, we assume that the OLT can only transmit a maximum of $L^{\max }$ packets per cycle. Hence, the number of groups $\frac{\lambda \bar{W}}{N L^{\max }}$ leads to additional reservation cycles with the limiting probability $P$, such that every cycle has $N \bar{V}$ reservations. Thus, we get:

$$
\Delta Y=\frac{\lambda \bar{W}}{N L^{\max }} \times P \times N \bar{V}=\frac{\lambda \bar{W} \bar{V}}{L^{\max }} \times P .
$$

Also note that, $L^{\max }=W^{\max } /(\bar{X} C)$. Thus, $\Delta Y=$ $\frac{\lambda \bar{W} \bar{V} \bar{X} C P}{W_{\max }}$, which ends the proof.

Theorem 4.1: Under UCS-based GBA, with limited service scheduling discipline, the mean downstream packet delay is determined by:

$$
\bar{W}=\frac{\lambda \overline{X^{2}}+(N+2-\rho) \bar{V}+(1-\rho) \sigma_{V}^{2} / \bar{V}}{2\left(1-\rho-\frac{(C \bar{V})^{2} \lambda \bar{X} \rho}{\left(W^{\max }\right)^{2}(1-\rho)}\right)} .
$$

Proof: Following Lemma 4.1, the mean reservation delay of a $N$-user reservation scheduling system under the limited service discipline, is given by:

$$
\bar{Y}=\frac{(N+1) \bar{V}}{2}+\frac{\lambda \bar{W} \bar{V} \bar{X} C P}{W^{\max }}
$$

From (1) and (6), we get:

$$
\bar{W}=\frac{\lambda \overline{X^{2}}+(N+2-\rho) \bar{V}+(1-\rho) \sigma_{V}^{2} / \bar{V}}{2\left(1-\rho-\lambda \bar{V} P \bar{X} C / W^{\max }\right)}
$$

and from (4) and (7), we get:

$$
\bar{W}=\frac{\lambda \overline{X^{2}}+(N+2-\rho) \bar{V}+(1-\rho) \sigma_{V}^{2} / \bar{V}}{2\left(1-\rho-\frac{(C \bar{V})^{2} \lambda \bar{X} \rho}{\left(W^{\max }\right)^{2}(1-\rho)}\right)}
$$

Remark 4.1: If $W^{\max }$ is sufficiently large, expression (8) approaches expression (2). This is reasonable because, in EPON systems, a limited service with a very large window size $W^{\max }$ converges to a gated service discipline.

Using Little's Law, the average size of queue $i$ at the OLT is given as follows:

$$
\overline{Q_{i}}=\lambda \bar{W} / N
$$




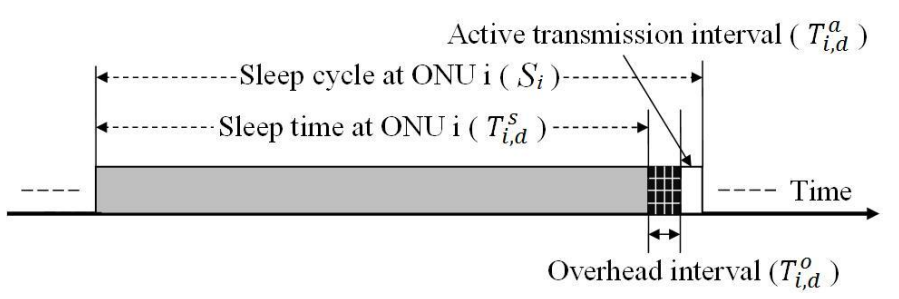

Fig. 4: Sleep cycle at ONU $i$ for the downstream traffic scheduling.

\section{A. Maximum ONU Sleep Time}

Let $E\left(X_{\mathrm{i}, \xi}\right)=\overline{X_{\mathrm{i}, \xi}}, E\left(X_{\mathrm{i}, \xi}^{2}\right)=\overline{X_{\mathrm{i}, \xi}^{2}}, \rho_{\mathrm{i}}=\lambda_{\mathrm{i}} \overline{X_{\mathrm{i}, \xi}}$, and $\lambda_{\mathrm{i}}=\lambda / N$. In steady-state, we have:

$$
\rho=\sum_{\mathrm{i}=1}^{\mathrm{N}} \rho_{\mathrm{i}}=\lambda \overline{X_{\mathrm{i}, \xi}}<1 .
$$

A diagram of the sleep cycle $S_{\mathrm{i}}$ under the downstream traffic scheduling for ONU $i$ is shown in Fig. 4. The sleep cycle is given as follows:

$$
S_{\mathrm{i}}=T_{\mathrm{i}, \mathrm{d}}^{s}+T_{\mathrm{i}, \mathrm{d}}^{o}+T_{\mathrm{i}, \mathrm{d}}^{a} .
$$

Packet, $\xi$, will be subject to a total delay $D_{\mathrm{i}, \xi}$, such that:

$$
D_{\mathrm{i}, \xi}(\xi)=W_{\mathrm{i}, \xi}(\xi)+T_{\text {prop }}+X_{\mathrm{i}, \xi}(\xi) .
$$

As $\xi \rightarrow \infty$, the expected total delay of a packet is given as follows:

$$
\overline{D_{\mathrm{i}, \xi}}=\overline{W_{\mathrm{i}, \xi}}+T_{\text {prop }}+\overline{X_{\mathrm{i}, \xi}},
$$

where $T_{\text {prop }}$ is the propagation delay [12]. From this expression, the maximum allowable packet delay can be expressed as

$$
\overline{W_{\mathrm{i}, \xi}} \leq \mathbb{D}_{\mathrm{i}, \xi}-T_{\text {prop }}-\overline{X_{\mathrm{i}, \xi}} .
$$

Hence, $\mathbb{D}_{\mathrm{i}, \xi}-T_{\text {prop }}-\overline{X_{\mathrm{i}, \xi}}$ is the upper bound of the expected packet delay $\overline{W_{\mathrm{i}, \xi}}$ in the system.

Theorem 4.2: Under UCS-based GBA, using the limited service discipline, the sleep time $T_{\mathrm{i}, \mathrm{d}}^{s}$, in which ONU $i$ can sleep during downstream transmissions, is determined by the following expression:

$$
T_{\mathrm{i}, \mathrm{d}}^{s}=S_{\mathrm{i}}-T_{\mathrm{i}, \mathrm{d}}^{o} \quad-\frac{\rho}{N} \times \frac{\lambda \overline{X_{\mathrm{i}, \xi}^{2}}+(N+2-\rho) \bar{V}+(1-\rho) \sigma_{V}^{2} / \bar{V}}{2\left(1-\rho-\frac{(C \bar{V} \rho)^{2}}{\left(W^{\max }\right)^{2}(1-\rho)}\right)} .
$$

Proof: Using the delay expression (8), the expected downstream waiting time for packet $\xi$ destined for ONU $i$ is given as:

$$
\overline{W_{\mathrm{i}, \xi}}=\frac{\lambda \overline{X_{\mathrm{i}, \xi}^{2}}+(N+2-\rho) \bar{V}+(1-\rho) \sigma_{V}^{2} / \bar{V}}{2\left(1-\rho-\frac{(C \bar{V} \rho)^{2}}{\left(W^{\max }\right)^{2}(1-\rho)}\right)} .
$$

By expressions (9) and (15), we have:

$$
T_{\mathrm{i}, \mathrm{d}}^{a}=\overline{Q_{\mathrm{i}}} \times \overline{X_{\mathrm{i}, \xi}}=\frac{\lambda \overline{W_{\mathrm{i}, \xi}} \overline{X_{\mathrm{i}, \xi}}}{N},
$$

which provides an expression for ONU $i$ 's reserved downstream data interval. From (11), (15), and (16), we get:

$$
\begin{aligned}
T_{\mathrm{i}, \mathrm{d}}^{s} & =S_{\mathrm{i}}-T_{\mathrm{i}, \mathrm{d}}^{o}-T_{\mathrm{i}, \mathrm{d}}^{a}=S_{\mathrm{i}}-T_{\mathrm{i}, \mathrm{d}}^{o} \\
& -\frac{\rho}{N} \times \frac{\lambda \overline{X_{\mathrm{i}, \xi}^{2}}+(N+2-\rho) \bar{V}+(1-\rho) \sigma_{V}^{2} / \bar{V}}{2\left(1-\rho-\frac{(C \bar{V} \rho)^{2}}{\left(W^{\max }\right)^{2}(1-\rho)}\right)},
\end{aligned}
$$

which ends our proof.

Finally, using the above analysis, we can extract the minimum sleep time for every ONU $i$ as follows:

$$
T_{\mathrm{i}, \mathrm{d}}^{\mathrm{s}, \min }=S_{\mathrm{i}}-T_{\mathrm{i}, \mathrm{d}}^{o}-\frac{\rho}{N} \times\left(\mathbb{D}_{\mathrm{i}, \xi}-T_{\text {prop }}-\overline{X_{\mathrm{i}, \xi}}\right) .
$$

Remark 4.2: The maximum ONU sleep time $T_{\mathrm{i}, \mathrm{d}}^{\mathrm{s}}$ is derived under the assumption that the downstream traffic is heavier than the upstream one. Nonetheless, in case the traffic on both directions is "balanced", the maximum sleep time based on the upstream traffic (such as the one in [4]) can be applied. Hence, the maximum ONU sleep time $T_{\mathrm{i}}^{\mathrm{s} \text {,max }}$ will then be set as the minimum between downstream-based and upstream-based values as follows:

$$
T_{\mathrm{i}}^{\mathrm{s}, \max }=\min \left(T_{\mathrm{i}, \mathrm{d}}^{\mathrm{s}}, T_{\mathrm{i}, \mathrm{u}}^{\mathrm{s}}\right)
$$

\section{Proposed Protocol for Energy-EfFicient SCHEDULING}

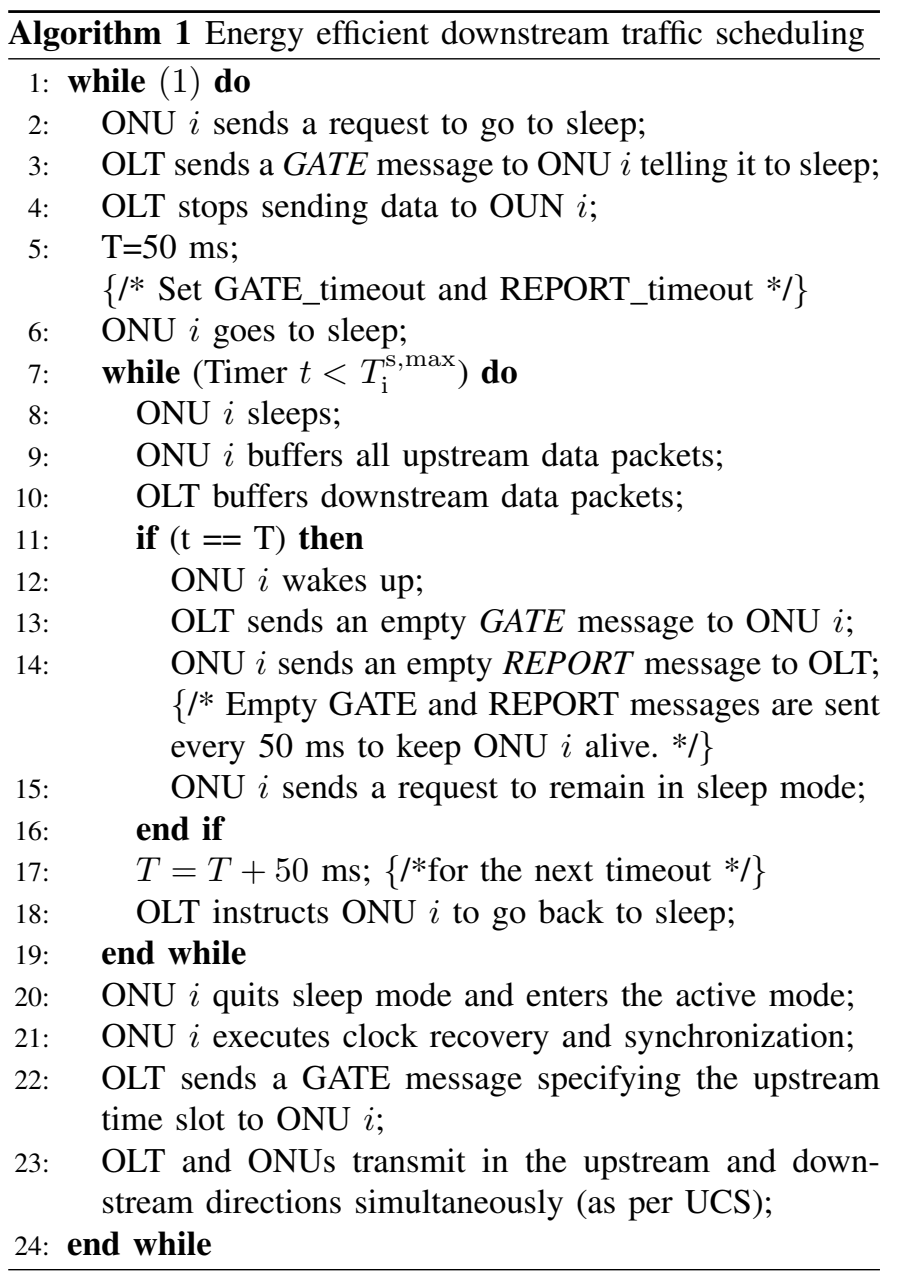

The pseudo-code of the proposed protocol for energyefficient scheduling under limited service discipline is given in Algorithm 1. To make the developed technique applicable to practical EPON systems, a scheduling protocol is provided in this subsection, aiming to achieve the maximum sleep time at each ONU according to the analysis result as in (19). It is understood that the proposed protocol should comply with MPCP (Multi-Point Control Protocol) which defines a timeout 
interval of $50 \mathrm{~ms}$ [13], after which an ONU will be fully deactivated. Thus, the proposed protocol has each ONU wake up every $50 \mathrm{~ms}$ just to exchange empty REPORT and GATE messages with the OLT in order to function properly.

As shown in Algorithm 1, all the downstream and upstream packets of ONU $i$ are firstly stored in the buffer at the OLT and ONU, respectively, within the sleep period of the ONU. Step 22 in the algorithm is arranged to transmit all the packets buffered at OLT and ONU $i$, immediately after the ONU quits sleeping and enters the active mode.

\section{Simulation AND Numerical Results}

In this section, simulations are conducted to verify the proposed analytical model for downstream traffic delay and demonstrate the effectiveness of the proposed scheduling algorithm. We are particularly interested in observing the impact on the scheduling performance due to the limited service discipline and the increase on the number of ONUs in the system. The section comprises three parts: (1) analytical model observation, (2) validation of the analytical model using simulations, and (3) performance measurement of presented scheme versus counterparts. MATLAB is used to simulate the proposed scheme and the scheduling algorithm in all three parts. In (2) and (3), the inter-arrival time of downstream packets destined for an ONU is generated according to an exponential distribution. A gated UCS-based upstream bandwidth allocation scheme is employed with a pre-defined GATE message size of $W^{\max }$. Each ONU has an equal share of the cycle time, $T_{\text {cycle }}$.

TABLE II: System Parameters

\begin{tabular}{|c|c|}
\hline Parameters & Description and Values \\
\hline$N$ & Number of ONUs: 8,16 and 32 \\
\hline$C$ & Line rate of downstream 1 Gbps [13] \\
\hline$G$ & Guard interval: $1 \mu s[13]$ \\
\hline$T_{\text {cycle }}$ & Cycle time $(\mathrm{N}=16): 2 \mathrm{~ms}[13]$ and $1.3 \mathrm{~ms}$ \\
\hline$W^{\max }$ & $\begin{array}{l}\text { Maximum transmission window size: } 15,500 \\
\text { bytes [13], 15,000 bytes, etc. }\end{array}$ \\
\hline $\bar{V}$ & Mean reservation interval: $1.512 \mu s$ \\
\hline$\overline{\overline{X_{\mathrm{i}, \xi}}}$ & Mean service time: $5.08976 \mu s^{1}$ \\
\hline$\overline{X_{\mathrm{i}, \xi}^{2}}$ & $\begin{array}{l}\text { The second moment of service time: } 51.1604 \\
(\mu s)^{2} 1\end{array}$ \\
\hline$T_{\mathrm{i}, \mathrm{d}}^{o}$ & $\begin{array}{l}\text { Overhead for downstream at ONU } i: 0.125 \\
\mathrm{~ms}[3]\end{array}$ \\
\hline
\end{tabular}

${ }^{1}$ : The values are calculated based on the data provided in [4].

\section{A. Analytical Model Observation}

The developed analytical model for the delay expression is examined using the system parameters given in Table II. In Fig. 5, it is clear that the average packet delay increases as the traffic load increases and when the number of ONUs increases; while the delay is almost the same regardless of the traffic load variation when the traffic load is very low.
Fig. 6 shows that with a typical number of ONUs and utilization factor $\rho$, the average packet delay increases as the transmission window size $W^{\max }$ decreases, which is under our expectation due to the system behavior of the limited service discipline (see the expression (15)). Although it can be observed via simulations, the optimal maximum transmission window size under the limited service discipline via the analytical derivation is obviously an interesting topic to explore, which is nonetheless left for future studies.

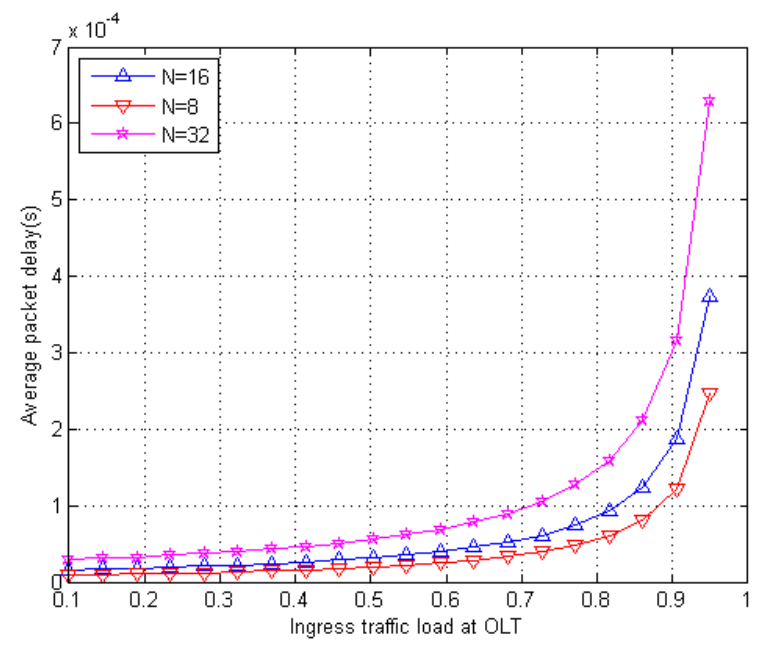

Fig. 5: Average Packet Delay with $W^{\max }=15,500$ bytes.

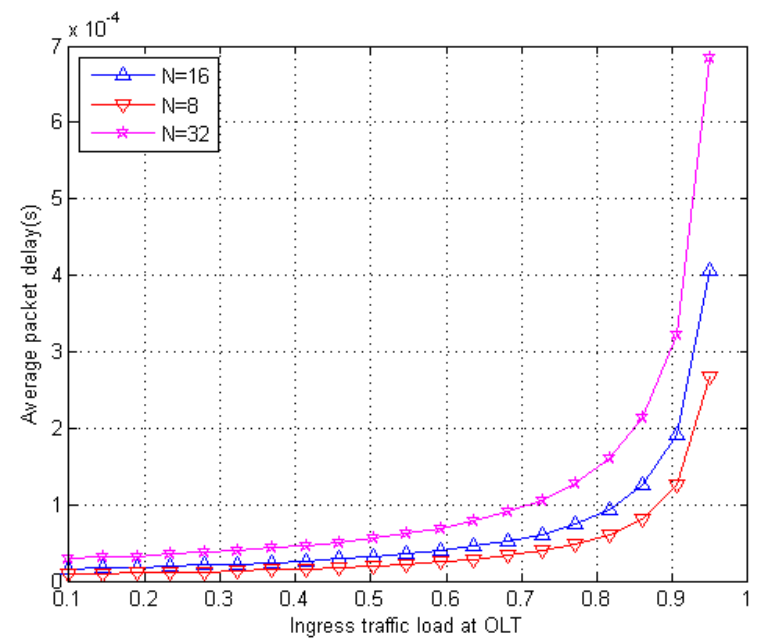

Fig. 6: Average Packet Delay with $W^{\max }=10,000$ bytes.

\section{B. Validation of Analytical Model}

The second set of experiments aims to validate the developed analytical model on the maximum sleep time of each ONU.

In these experiments, variable bit rate (VBR) and best effort (BE) packets have a payload size that varies from 64 to 1518 bytes with the distribution based on [14] as follows: 64 bytes (47\%), 300 bytes (5\%), 594 bytes (15\%), 1300 byte(5\%), 
and 1518 bytes (28\%). Fig. 7 and Fig. 8 verify the derived maximum sleep time on different ingress traffic loads via simulations. Fig. 7 examines the scenarios of different window sizes (14,500 bytes, 15,000 bytes, and 15,500 bytes) with 16 ONUs in the system while Fig. 8 emphasizes on the impacts of the different number of ONUs in the system as well as the different transmission window size.

Firstly, we can see a close match between the simulation and analytical results, which validates the proposed sleep time analysis. Fig. 7 demonstrates that with increased ingress traffic load for each ONU or the decreased window size, the ONU sleep time gets shorter. On the other hand, Fig. 8 shows that the maximum window size imposes noticeable effects on the maximum allowed ONU sleep time, where a larger window size yields longer sleep time. This meets our expectation and understanding on batch mode transmission, in which a large transmission window allows each ONU to have smaller percentage of overhead due to switching between active and sleep modes. Note that this figure also implies that there is a minimum sleep time at a certain window size subject to the upper bound of packets' delay when the traffic load is very high during the downstream traffic scheduling.

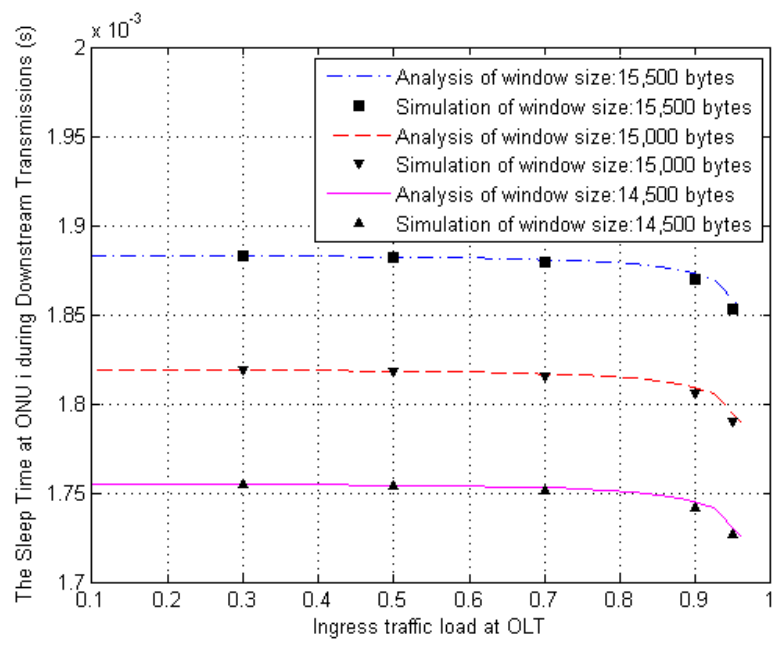

Fig. 7: Sleep time versus ingress load for each ONU.

\section{Comparison with Counterparts}

In this section, we compare the proposed scheduling algorithm with a legacy active DBA (i.e., no ONU sleep), and the Just-in-time (JIT)-based IPACT [8], in terms of energy consumption. Assuming that the downstream traffic load on each ONU is sufficiently larger than that of the upstream, the allowed sleep time duration of each ONU is indifferent of the upstream traffic load under the UCS approach. The energy consumption of an ONU can be calculated by the following expression [6]:

$$
E_{\mathrm{ONU}}=T_{\text {active }} \times P_{\text {active }}+T_{\text {sleep }} \times P_{\text {sleep }}
$$

where $P_{\text {active }}$ and $P_{\text {sleep }}$ denote the active power consumption and the sleep power consumption respectively. Note that

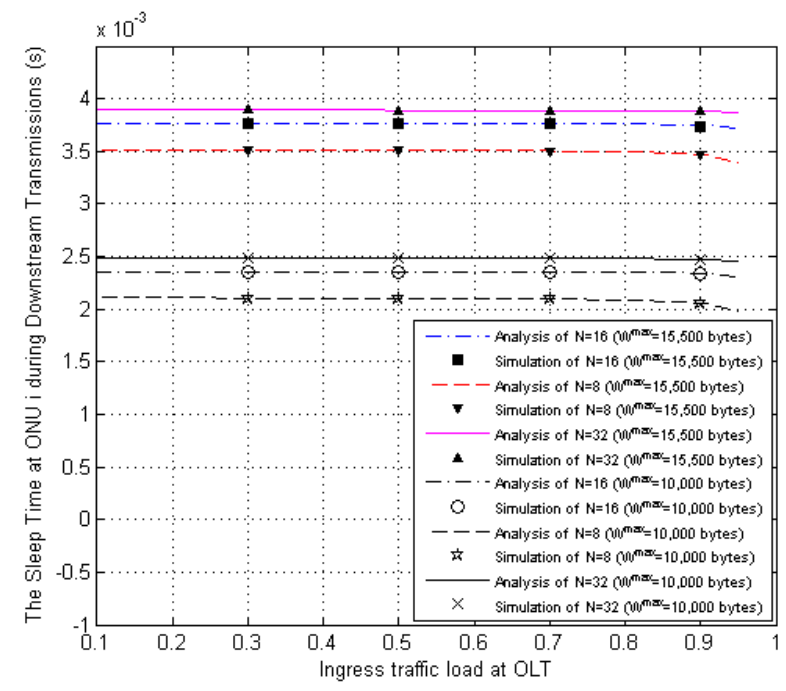

Fig. 8: Sleep time versus ingress load for each ONU.

$T_{\text {active }}$ includes the overhead time in the formula. In the comparison we take $P_{\text {active }}$ as $3.85 \mathrm{~W}$ and $P_{\text {sleep }}$ as $0.75 \mathrm{~W}$ during deep sleep [3].

Fig. 9 shows the energy consumption for different numbers of ONUs under the maximum transmission window of 15,500 bytes over a 10 minutes simulation run-time. Note that under IPACT, each ONU can only sleep based on the DBA at the OLT, and may be switched to active at random times depending on the scheduled transmission slot. As observed, the energy consumption of an ONU without sleeping is 2310 Joules while the energy consumptions of 8,16 , and 32 ONUs in the system are approximately 295, 235, and 207 Joules respectively, depending on the traffic load. The figure also shows that the proposed downstream traffic scheduling scheme can achieve approximately $90 \%$ reduction in energy consumption compared with the scheme without sleep mode. On the other hand, the JIT-based IPACT scheme can only achieve a reduction up to $60 \%-70 \%$ as shown in the figure. All these results clearly demonstrate the merits of our proposed downstream scheduling protocol in being pushed into practical EPON deployments.

\section{CONCLUSIONS}

This paper presents a novel downstream traffic scheduling protocol for energy conservation in EPONs. The proposed scheme is based on a new analytical model in which we analyze the expected downstream packet delay and the ONU sleep time under the Green Bandwidth Allocation (GBA) framework. To the best of our knowledge, our model is the first analytical study that investigates the maximum ONU sleep time under downstream-based delay constraints, which is expected to perfectly meet the practical EPON system requirement. Simulation results highlight the advantages of the proposed scheme and provide insights to the relation between maximum transmission window size and maximum ONU sleep time. Results also show that, unlike existing energy saving schemes in EPON, our scheme is able to ensure almost $90 \%$ 


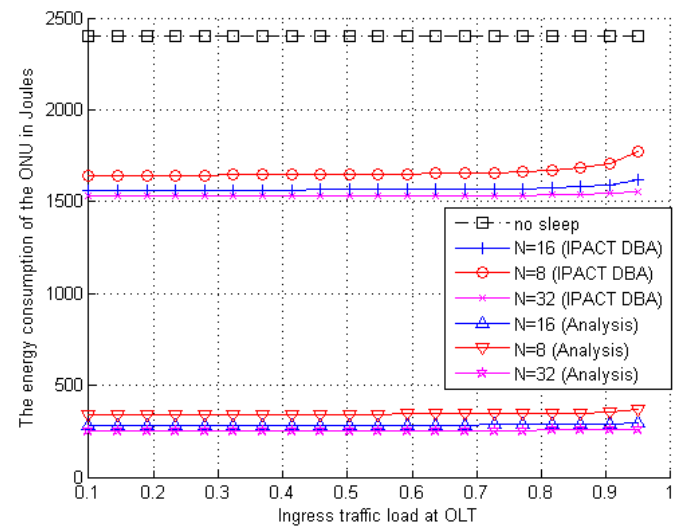

Fig. 9: The energy consumption of the ONU in Joules $\left(W^{\max }=\right.$ $15,500$ bytes $)$.

reduction in the ONU energy consumption, while maintaining the QoS requirements for both the upstream and downstream traffics.

\section{REFERENCES}

[1] J. Zhang and N. Ansari, "Towards energy-efficient 1G-EPON and 10 GEPON with sleep-aware MAC control and scheduling," IEEE Communications Magazine, vol. 49, no. 2, Feb. 2011.

[2] Y. Yan, S.-W. Wong, L. Valcarenghi, S.-H. Yen, D. R. Campelo, S. Yamashita, L. Kazovsky, and L. Dittmann, "Energy Management Mechanism for Ethernet Passive Optical Networks (EPONs)," Proceedings of IEEE International Conference on Communications(ICC'10), Cape Town, South Africa, May 2010.

[3] A. R. Dhaini, P.-H. Ho, and G. Shen, "Toward Green Next-Generation Passive Optical Networks," IEEE Communications Magazine, Vol. 49, Issue 11, pp 94-101, Nov. 2011.

[4] A. R. Dhaini, P.-H. Ho, and G. Shen,"Energy Efficiency in Ethernet Passive Optical Networks: For How Long Can ONU Sleep?" University of Waterloo, Tech. Rep., Mar. 2011. [Online]. Available: https://ece. uwaterloo.ca/ adhaini/Files/G_EPON_Sleep.pdf.

[5] S. Bharati and P. Saengudomlert, "Analysis of Mean Packet Delay for Dynamic Bandwidth Allocation Algorithms in EPONs", IEEE/OSA Journal of Lightwave Technology, 28(23):3454-3462, Dec. 2010.

[6] S. Wong, L. Valcarenghi, S. Yen, D. R. Campelo, S. Yamashita, and L. Kazovsky, "Sleep Mode for Energy Saving PONs: Advantages and Drawbacks," IEEE GLOBECOM'09, Hawaii, USA, Dec. 2009.

[7] S. Wong, S. Yen, P. Ashfar, S. Yamashita, and L. Kazovsky, "Demonstration of Energy Conserving TDM-PON with Sleep Mode ONU Using Fast Clock Recovery Circuit," OFC/NFOEC'10, March 2010.

[8] L. Valcarenghi, S.-W. Wong, D. R. Campelo, S.-H. Yen, S. Yamashita, P. G. Raponi, L. Kazovsky, and P. Castoldi, "Energy Efficiency in Optical Access Networks," in Italian Networking Workshop, Cavalese, Italy, Jan. 2011.

[9] S.S.W. Lee, A. Chen, "Design and Analysis of a Novel Energy Efficient Ethernet Passive Optical Network," IEEE ICN, pp.6-9, Sep. 2010.

[10] G. Kramer and B. Mukherjee, "Interleaved Polling with Adaptive Cycle Time (IPACT): Protocol Design and Performance Analysis," Tech. rep. CSE-2001-4, Dept. of Comp. Sci., UC Davis, Aug. 2001.

[11] D. Bertsekas and R. Gallager, Data Networks, Prentice-Hall, 1992.

[12] C. Lam, Passive Optical Networks, Academic Press (ELSEVIER), 2007.

[13] G. Kramer, Ethernet Passive Optical Networks, New York: Mc-Graw Hill, 2005.

[14] G. Kramer, B. Mukherjee, S. Dixit, Y. Ye, and R. Hirth, "Supporting Differentiated Classes of Service in Ethernet Passive Optical Networks," OSA Journal of Optical Networking (JON), vol. 1, no. 8/9, pp. 280-298, Aug. 2002. 